\title{
Le don de colostrum en situation de séparation mère-enfant
}

De l'aliment au traitement

\section{Céline Verguet}

\section{OpenEdition}

\section{Journals}

Édition électronique

URL : https://journals.openedition.org/tc/8819

DOI : $10.4000 /$ tc. 8819

ISSN : 1952-420X

\section{Éditeur}

Éditions de l'EHESS

Édition imprimée

Date de publication : 17 mai 2018

Pagination : 168-179

ISBN : 978-2-7132-2750-9

ISSN : 0248-6016

\section{Référence électronique}

Céline Verguet, «Le don de colostrum en situation de séparation mère-enfant », Techniques \& Culture [En ligne], 69 | 2018, mis en ligne le 17 mai 2020, consulté le 29 septembre 2022. URL : http:// journals.openedition.org/tc/8819; DOI : https://doi.org/10.4000/tc.8819 


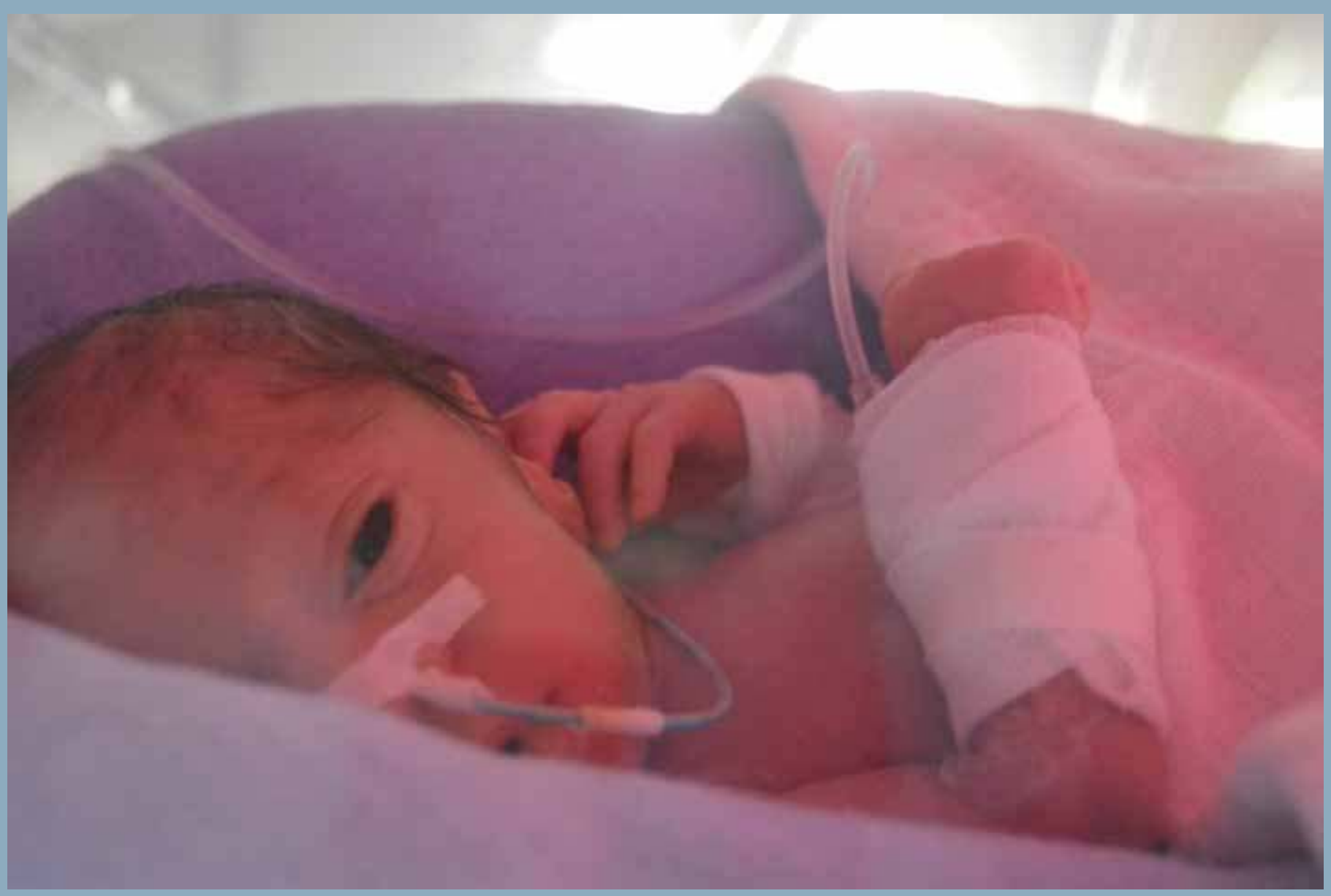




\section{Le don de colostrum en situation de séparation mère-enfant}

\section{De l'aliment au traitement}

Premier fluide émis par la glande mammaire pendant 3 jours après l'accouchement, le colostrum est un apport hydrique, nutritionnel, immunoprotecteur et enzymatique régulier pour le nouveau-né (Candau et al. à paraître). Chez tous les mammifères, il est produit alors que la glande mammaire est en transition sous l'effet conjugué des changements physiologiques maternels qui surviennent après la parturition, et de la stimulation par le petit. En 2013, des chercheurs en immunologie et en anthropologie commencent à l'Hôpital l'Archet de Nice une étude inscrite dans le cadre d'un programme international de recherche inédit sur la toute première phase de l'allaitement. Coordonné par Joël Candau et financé par l'ANR (2013-2016), ce programme, conduit dans 7 pays situés sur 4 continents $^{1}$, a été consacré au don et à la consommation néonatale du colostrum ainsi qu'à la compréhension des logiques qui les régissent.

La présentation des premiers résultats aux soignants de l'hôpital impliqué dans la problématique de la séparation parents/enfant lors d'une naissance prématurée a favorisé l'élaboration inédite d'un double protocole de soin médical et infirmier consistant à donner le colostrum de leur mère à des enfants nés en dessous de 32 semaines d'aménorrhée. La mère exprime son colostrum dans le tube d'une seringue qui lui est fournie et préalablement bouchée puis l'obture avec le piston une fois la substance à l'intérieur. Ce nouveau protocole nécessite que le colostrum soit recueilli puis transporté du sein de la mère à la bouche de l'enfant grand prématuré pour un don artificiel au coton-tige ou à la seringue. Il engage souvent mais pas systématiquement la conservation du liquide. Comme il n'existe pas de recommandations propres à cette pratique, les soignants suivent celles d'« hygiène pour la préparation et la conservation des biberons » de l'Agence française de sécurité sanitaire des aliments (AFSSA, 2005)² prévues pour le lait de femme et de mère délivré en biberon. Ainsi, plusieurs manipulations, plusieurs protagonistes - soignants ou famille -, plusieurs lieux de conservation comme le réfrigérateur de la nurserie ou ceux de la biberonnerie interviennent jusqu'à l'absorption par l'enfant. Mal ou pas 
conservé, il est parfois jeté. Ce don de la mère à son bébé grand prématuré, aussi protocolaire et médicalement assisté soit-il, est en fait un bricolage élaboré dans le quotidien de pratiques professionnelles et de maternage.

Cet article s'appuie sur des données recueillies de manière informelle uniquement auprès des soignants après l'enquête ethnographique conduite en France au sein des services de néonatologie et de maternité de l'Archet. Il porte sur les manières dont le colostrum est exprimé, conservé puis donné dans la situation où la mère est séparée de son enfant grand prématuré. Le questionnement ici est annexe à l'ANR. Détaché de sa problématique, il est fondé sur le constat de l'impact majeur de l'enquête sur le terrain même de sa conduite. Quel est le rôle que l'on fait jouer au colostrum dans les soins apportés à ces enfants? La technicisation du don a-t-elle un impact sur la substance elle-même et ses représentations dans le cadre médical? Je commencerai par resituer le questionnement de l'article dans le cadre plus large du projet ANR et de ce qu'il a favorisé: la mise en place d'un protocole de soin spécifique et le développement de nouvelles pratiques autour de la santé des nouveau-nés séparés de leur mère. Ensuite, je décrirai le parcours du colostrum du sein de la mère à la bouche du bébé et plus particulièrement les techniques d'expression et de conservation qui conduisent au don indirect de cette « gelée royale ${ }^{3} »$. Mon objectif est de mettre en lumière ce que la situation de don de colostrum aux grands prématurés durant cette courte période implique de spécifique, en particulier en termes de pratiques d'allaitement et de pratiques professionnelles des soignants.

\section{De l'ANR Colostrum au " projet colostrum "}

Lait mature (droite), colostrum (gauche)

\section{Une étude inédite à l'impact inattendu}

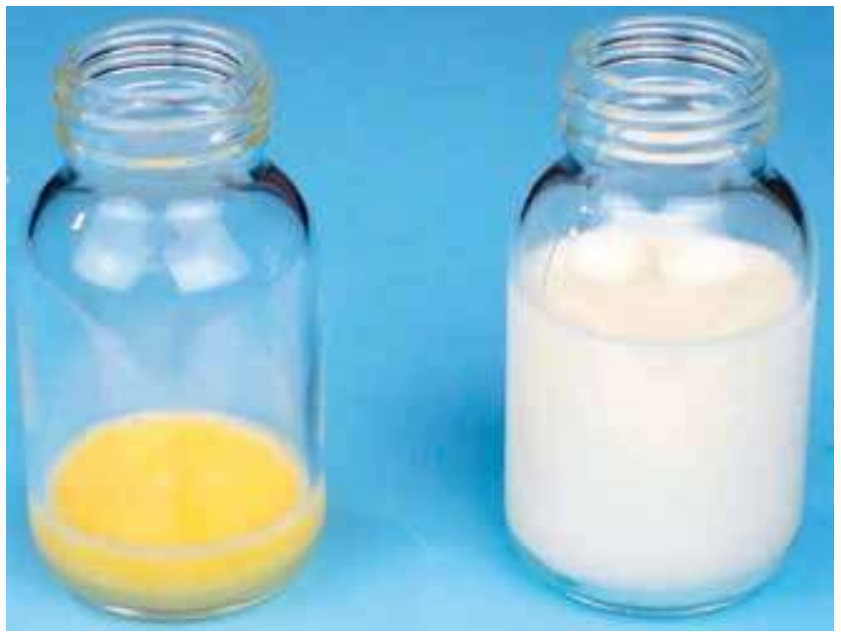

L'ANR Colostrum comprend deux volets complémentaires, l'un anthropologique et l'autre biologique, celui-ci structuré autour de deux axes, psychobiologique et immunologique. Le premier volet vise à documenter les pratiques et les représentations relatives à la consommation néonatale du colostrum et à identifier les variables sociales et culturelles susceptibles d'expliquer la consommation ou non de cette substance ${ }^{4}$. En effet, les données sur le don et la consommation de colostrum sont bien plus rares que celles sur l'allaitement maternel en général. Le deuxième volet s'attache à identifier les propriétés fonctionnelles, sensorielles et immunologiques. En outre, un volet open science a été mis en place pour la restitution des résultats ${ }^{5}$. 
La mise en œuvre de cette étude à la maternité et en néonatalogie et la communication des premiers résultats de l'enquête anthropologique et de l'étude immunologique ont impulsé, au sein de l'hôpital, un projet de recherche concerté appelé «projet colostrum» (Verguet et al. 2016). Il a conduit des personnels des services de maternité et de néonatalogie pourtant indépendants et spatialement éloignés les uns des autres à se pencher sur l'intérêt du colostrum pour les nouveau-nés prématurés et sur l'optimisation de son recueil par expression manuelle chez les mères. Dans les pratiques et les discours de soignants, la phase colostrale n'était pas spécifiquement différenciée de l'allaitement. La conséquence, particulièrement dans les unités de médecine néonatale, était un don de colostrum souvent «négligé » signale Sandrine ${ }^{6}$. D’ailleurs, une étude menée dans le service de réanimation néonatale entre octobre 2012 et mai 2014 sur les nouveau-nés prématurés de poids de naissance inférieur à $1200 \mathrm{~g}$ montre que sur 49 enfants de mères souhaitant allaiter, seuls 16 \% recevaient du colostrum de leur mère ${ }^{7}$.

\section{Le protocole de soin}

La formalisation de ce projet passe par la mise en place d'un double protocole de soin.

\section{Le protocole médical}

Il est porté par le Docteur Stéphanie De Smet ${ }^{8}$, avec l'installation d'un essai clinique ${ }^{9}$ sur l'«impact de l'administration oropharyngée du colostrum dans les 48 premières heures de vie chez le nouveau-né prématuré de terme inférieur à 32 semaines d'aménorrhées ${ }^{10} »$. Il consiste à lui administrer le colostrum de sa mère par «tapissage» de la cavité buccale dans les 6 heures qui suivent la naissance. L'objectif est d'évaluer l'impact du colostrum sur la tolérance digestive, l'immunité et la croissance staturo-pondérale à court terme et à 1 an. Deux groupes d'enfants sont comparés: le groupe colostrum suivant strictement le protocole et le groupe témoin auquel est donné du lait de lactarium selon la même modalité, la même durée et en même quantité. En outre, tous les nouveau-nés suivent un protocole de nutrition entérale identique mis en place dans les premières heures de vie et sur la durée de l'essai clinique avec du lait cru provenant de la mère ou du lait de lactarium en cas d'allaitement non désiré ou de quantité considérée comme trop faible. Dans le cadre du protocole, le don de colostrum des mères à leur enfant grand prématuré se fait sur prescription médicale et après qu'elles aient signé le consentement éclairé relatif à l'étude.

\section{Le protocole infirmier}

Il passe par le développement d'outils d'éducation thérapeutique pour «sensibiliser au colostrum et à ses bienfaits ${ }^{11} »$ et pour le déploiement de la pratique du recueil et du don précoce en salle de naissance à destination des grands prématurés ${ }^{12}$. Conduit par les 3 consultantes en lactation, il consiste à améliorer l'accompagnement des mères de ces enfants en anténatal et/ou dans les heures qui suivent l'accouchement pour l'expression de leur colostrum.

La procédure médicale commence le plus souvent en anténatal avec la prise en charge des futures mères hospitalisées en grossesse à risque élevé. Un pédiatre ou une infirmière puéricultrice 
prend alors connaissance du projet parental d'allaitement. Au cours de cette visite, les mères sont avisées de la possibilité d'allaiter ou non leur nouveau-né, même en cas de séparation et de grande prématurité. Si cette situation survient et que la mère ne souhaite pas allaiter, on lui explique que son bébé recevra du lait provenant de lactarium et non du lait industriel. Mais quelle que soit la décision de la mère quant au mode d'allaitement en cas de séparation (son propre lait dit « lait cru» ou de lactarium), le don de colostrum est systématiquement proposé. Lorsque la mère refuse, elle est sollicitée pour l'inclusion dans le groupe témoin.

Une fiche a été conçue afin d'expliquer aux mères les étapes à suivre pour « faciliter le recueil de colostrum». Elle est également utilisée pour former les équipes de soin de tous les services impliqués dans le protocole d'accompagnement des mères dans leur apprentissage des gestes favorisant la « récolte ${ }^{13} »$ de la substance. Elle a été élaborée à partir des travaux de la pédiatre Jane Morton de l'université de Stanford qui font la démonstration de l'intérêt d'employer l'expression manuelle afin d'optimiser le recueil du colostrum et la mise en route de la lactation ${ }^{14}$. Dans les situations de mise au sein d'un bébé né à terme et sans problème de santé, la quantité de colostrum dans une tétée est comprise entre 2 à $6 \mathrm{ml}$. Il n'est donc pas possible pour les mères séparées de leur enfant d'utiliser un tire-lait électrique ou manuel, car la faible quantité de liquide serait irrécupérable, coincée dans le tuyau. L'expression manuelle est la seule à convenir à la situation. De plus, la naissance d'un enfant prématuré place l'allaitement maternel face à une multitude de barrières physiologiques, entre autres la séparation, la césarienne, la non-maturité de succion nutritive du bébé ou son équipement médical. Le volet infirmier vise à ce que le projet d'allaitement maternel ne soit plus relégué au second plan ni sa mise en route retardée - impliquant l'impasse faite sur le colostrum -, voire abandonnée.

Si dans les services de réanimation néonatale en France, donner le colostrum de leur mère aux enfants grands prématurés est une pratique émergente, l'enquête internationale a fait remonter qu'elle est courante en Allemagne alors qu'elle est obligatoire au centre hospitalier universitaire German Urquidi de Cochabamba en Bolivie ${ }^{15}$. Par ailleurs, d'autres essais cliniques conduits dans le domaine de la pédiatrie s'intéressent aux effets et éventuels bénéfices du don de colostrum en oropharyngée sur les enfants nés avant terme. Snyder et al. (2017) montrent par exemple qu'outre les bénéfices du lait maternel apporté par le colostrum à ces nouveau-nés, ce protocole de don peut servir de catalyseur pour augmenter les taux d'allaitement et leur durée chez les prématurés. L'hôpital l'Archet est le seul établissement de la région Provence Alpes Côte d'Azur à l'avoir mis en place. 


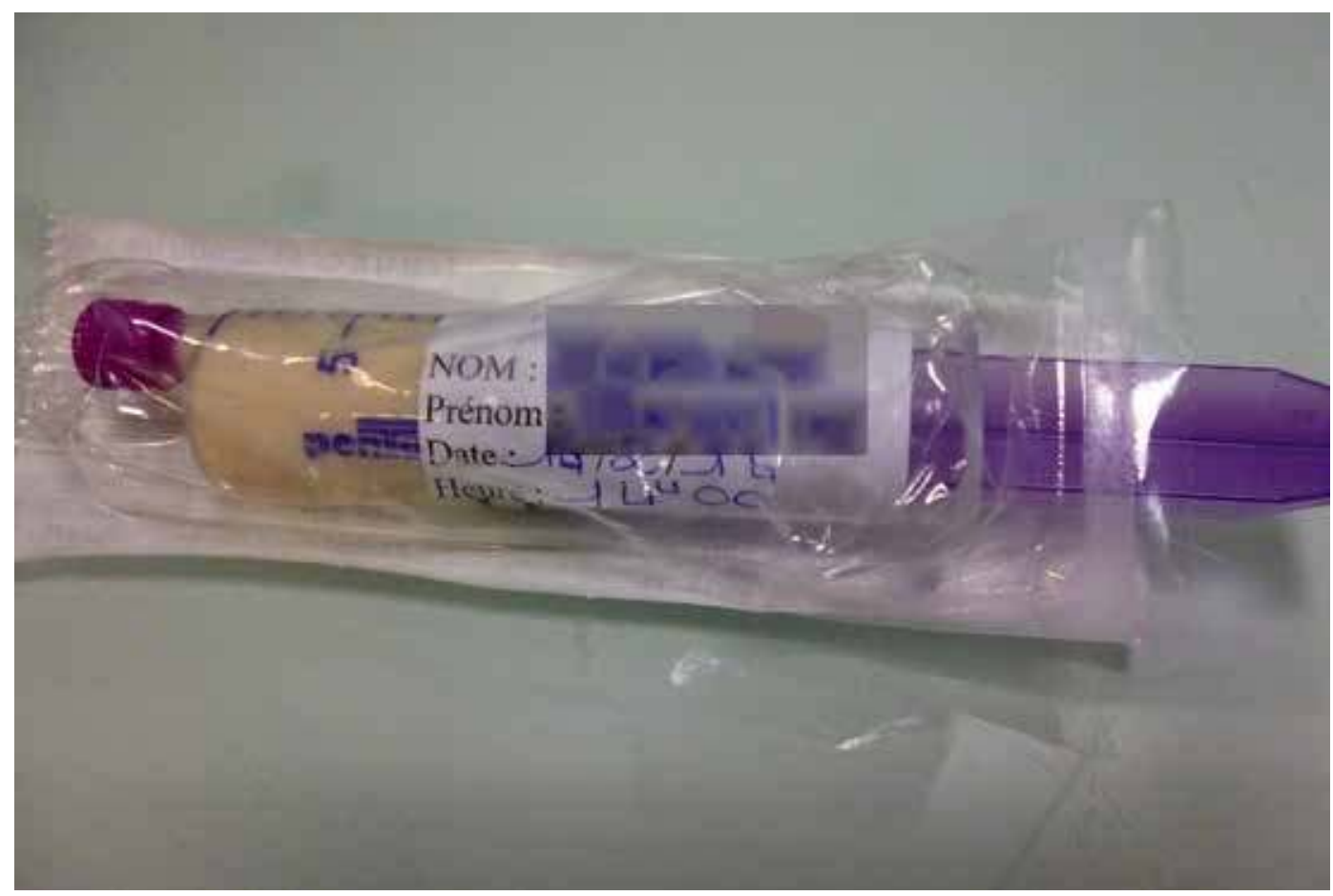

2. Colostrum réservé dans une seringue

\section{Du sein de la mère à la bouche du bébé}

\section{Expression du colostrum}

Depuis la mise en place du protocole courant 2014, c'est généralement en salle de naissance que les soignants proposent aux mères d'exprimer le colostrum dans les six premières heures ${ }^{16}$ après la naissance puis aussi fréquemment que si elles devaient mettre leur enfant au sein à chacune des phases d'éveil, soit 7 à 8 fois en 24 heures. Parfois, c'est l'équipe de médecine néonatale qui s'en charge en venant donner des nouvelles du bébé. L'acceptation des mères est qualifiée de «phénoménale» par l'interne en charge de l'étude. Selon Sandrine, déjà dans la phase préliminaire au démarrage de l'essai clinique, non seulement le nombre de dons aux grands prématurés avait atteint $68 \%$ à l'été 2015 mais les mères recueillaient désormais du colostrum jusqu'à 5 fois par jour contre 0 à 1 fois avant le début de l'étude.

Sur la fiche qui accompagne les mères dans leur recueil de colostrum, il est expliqué que «même si votre bébé n'est pas alimenté, votre lait sera utilisé pour les soins de réassurance ». Cette dernière expression, si elle reste «technique» et peu appropriable par des profanes, engage au moins les représentations associées aux deux termes qui accordent une connotation 
positive au fait de donner son colostrum. À bien y regarder, allaiter, que ce soit au sein ou en don indirect, n'est jamais vraiment une décision individuelle. C'est une pratique qui n'échappe pas tant aux injonctions sociales que médicales. Elle ne peut pas s'effectuer indépendamment d'un ensemble de prescriptions ou de représentations morales et symboliques en circulation au sein de la société (Gojard 2003).

Contrairement au lait cru, le traitement du colostrum, de son recueil à son application oropharyngée, est uniquement manuel. Ce sont d'abord les mains des soignants qui montrent. Au-delà de la méthode décrite sur la fiche distribuée, ils transmettent un savoir et un savoirfaire haptique généralement par une démonstration sur des seins en laine prévus à cet effet ou sur leur propre poitrine ou encore en prenant les mains de la patiente pour la guider,

. Expression du colostrum. Démonstration à l'aide d'un sein en laine

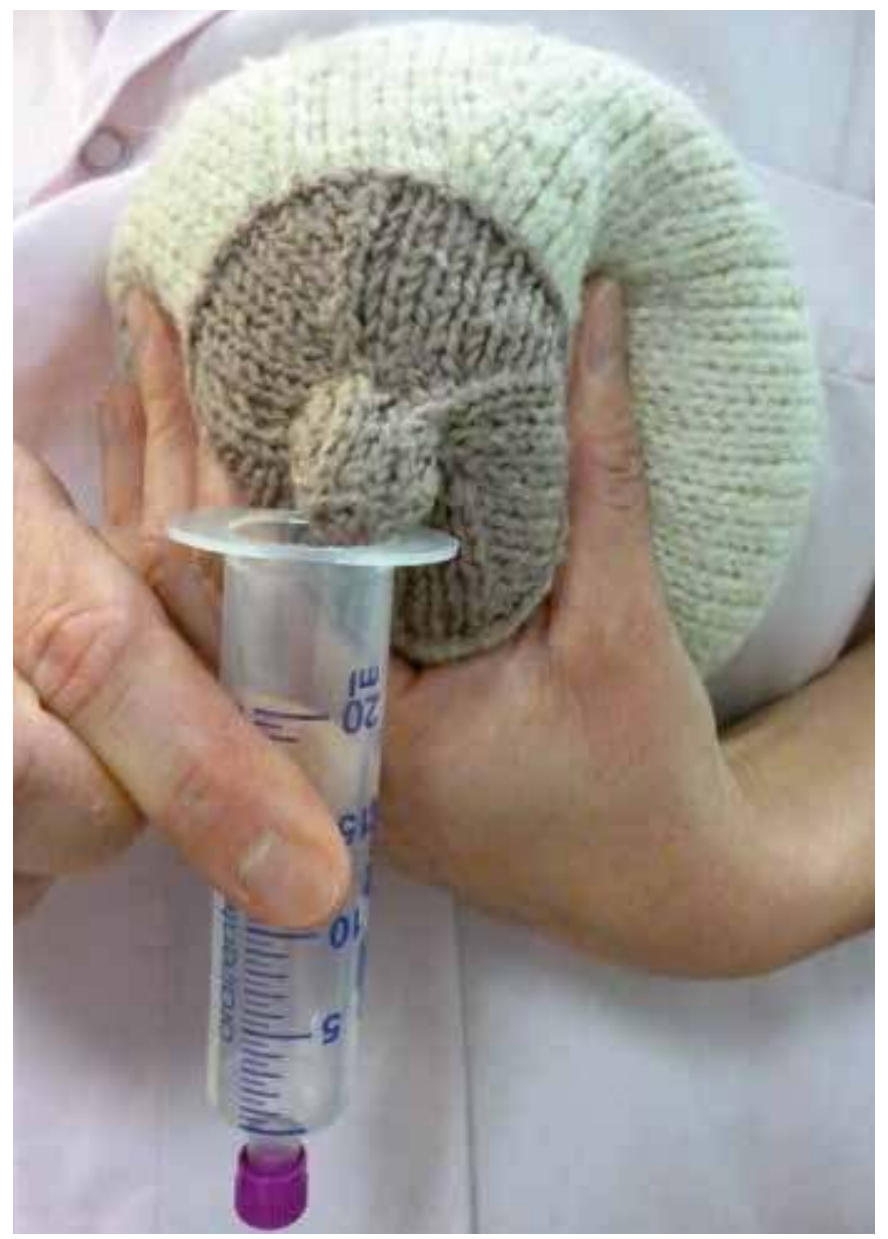

avec son accord, dans ses gestes. Cette dernière méthode semble la plus efficace pour l'acquisition de données kinestésiques (e.g. pression, douleur) qui vont participer à provoquer l'effet physiologique escompté : l'écoulement du premier lait. Les mères sont ensuite encouragées à reproduire «par mimétisme» (Chloé) ce qu'elles ont vu, entendu et senti, elles vont rechercher les modalités sensorielles (Sola 2015) qui les conduiront seules à faire perler le colostrum.

La première expression manuelle du colostrum a lieu soit en salle de naissance ou dans la salle de réanimation néonatale auprès de l'enfant, soit dans sa chambre en maternité, quatre étages au-dessus, où la mère est envoyée deux heures post-partum si elle ne rencontre aucune complication. «La moindre goutte est immédiatement amenée pour le bébé. Le père peut venir lui donner» (Chloé). La préciosité attachée au liquide est liée à sa faible quantité et à la courte durée de sécrétion, autrement dit à sa rareté, mais aussi aux effets qui lui sont prêtés tant du point de vue de l'idéologie professionnelle que des convictions personnelles des soignants. Les discours des mères qui connaissent le colostrum sont systématiquement des emprunts au discours médical, plutôt sous la forme d'une adhésion qu'une véritable appropriation. C'est ce qu'a montré l'enquête ANR (Candau \& Verguet à paraître). 


\section{La conservation du colostrum}

Comme il n'existe pas de méthode de conservation spécifique au colostrum, «on suit le protocole AFSSA 2005, les recommandations sur la conservation du lait maternel (Sandé). Il y est écrit que «les conditions de recueil et de conservation du lait de femme doivent être adéquates, conformes aux règles d'hygiène». Même si le colostrum et le lait (de transition ou mature) diffèrent tant en quantité qu'en qualité (macro- et micronutriments, principes immunitaires, facteurs de croissance, composants minéraux), ce liquide est considéré comme du lait, le premier adapté à l'âge du bébé ${ }^{17}$. Il est parfois décrit, tant par les soignants que par les mères, comme « un lait concentré». Quant au matériel utilisé pour le recueil et la conservation de la substance, soit il a été détourné de son usage premier et choisi pour sa fonctionnalité et son ergonomie dans le stock de marchandises disponibles - c'est le cas de la seringue qui sert de réceptacle, de contenant hermétique de stockage et de transport mais qui permet aussi de délivrer la substance directement dans la bouche du bébé; soit sélectionné pour son opérativité sur d'autres substances pensées comme proches du colostrum (lait cru ou de lactarium, médicament) - c'est le cas du réfrigérateur.

Les enfants du groupe colostrum ont reçu de 1 à 12 doses - terme employé dans l'essai clinique - sur la durée du protocole. La mère ou le père ${ }^{18}$ peuvent eux-mêmes descendre le liquide recueilli en réanimation néonatale et l'inoculer ${ }^{19}$ à leur enfant. Lorsqu'ils sont présents ensemble ou séparément d'un bout à l'autre de la chaîne, les sollicitations orales sont presque toujours faites à base de «colostrum fraîchement exprimé» (Chloé). La substance est considérée comme telle dans un laps de temps de 4 heures « entre le début du recueil et la fin de l'utilisation (fin de la consommation du biberon) de ce lait laissé à température ambiante» (AFSSA). S'il est amené à être conservé au frais, c'est que la chaîne parents-enfant a dû être interrompue par l'intervention d'un soignant: "C'est l'équipe d'en haut qui le place dans le frigo. À la pouponnière, c'est le frigo qui est fait pour recevoir les médicaments qui est utilisé» (Sandrine).

Si certaines doses ont été délivrées directement après avoir été exprimées - c'est très souvent le cas de la première -, d'autres ont nécessité d'être conservées soit comme on vient de le voir «à l'air ambiant» (Chloé), sur la table de nuit par exemple, soit dans un réfrigérateur pour pousser la durée de conservation à 48 heures en attendant que «quelqu'un le descende» (Chloé). Ces conditions de conservation sont plus encore valables pour «les enfants les plus à risque comme les grands prématurés. [...] Quand le nourrisson est né à terme, sans antécédents, et qu'il est en bonne santé et nourri au

4. Conservation de lait maternel dans le réfrigérateur de la pouponnière (service de la maternité)

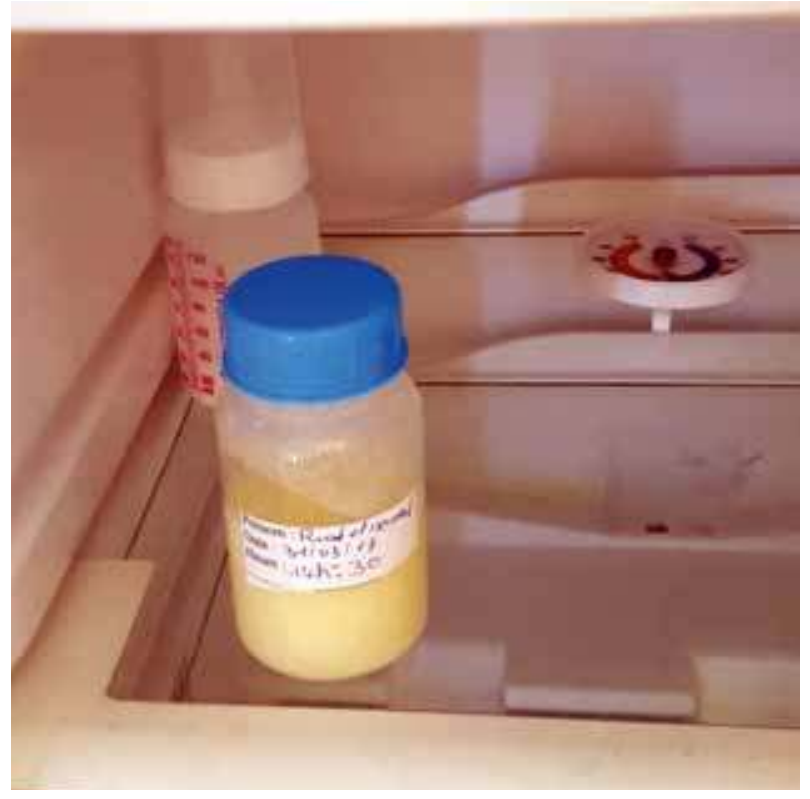




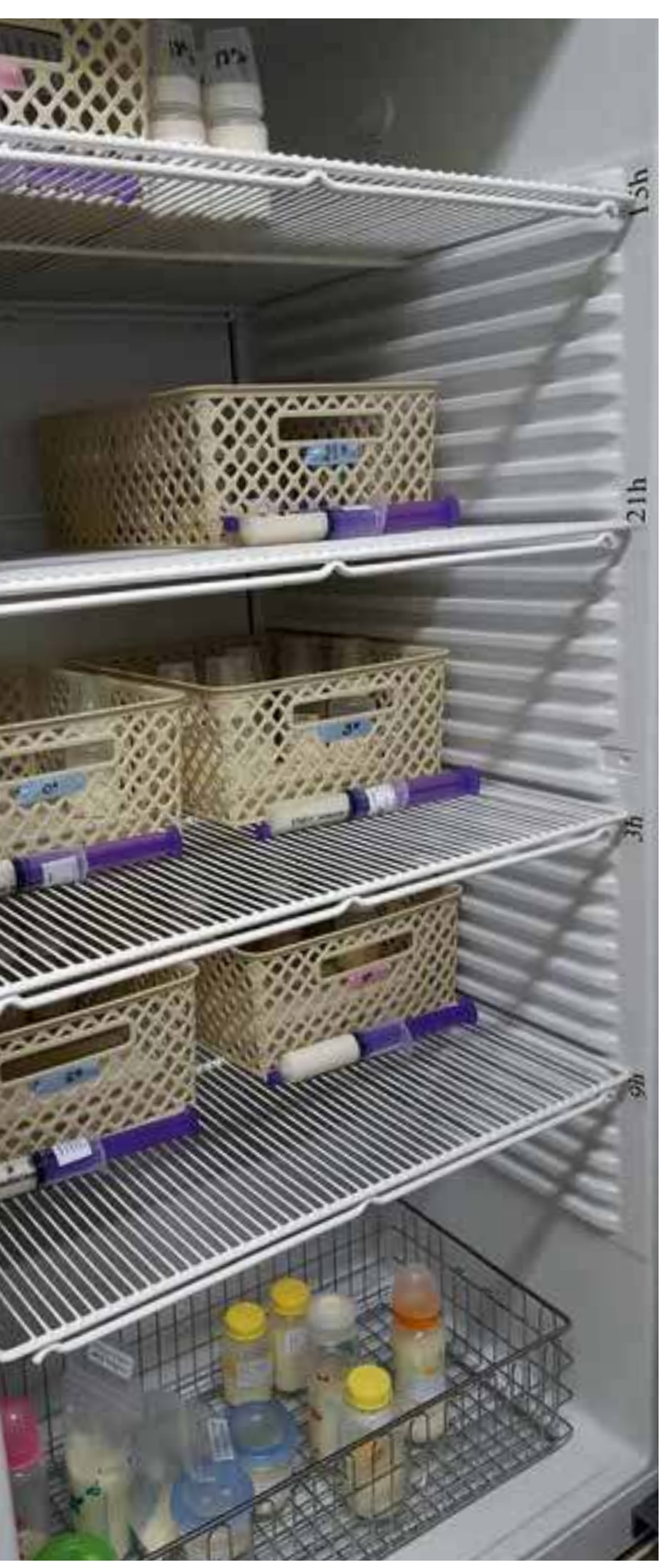

5. Conservation du lait dans une biberonnerie (service de néonatalogie) domicile, le risque pour la santé de l'enfant lié à une éventuelle contamination est estimé faible » (AFSSA). Si l'on comprend le fait qu'il s'agit d'éviter la contamination biologique, rien dans ce document n'explique les délais fixés à 4 et 48 heures. Mais ce sont des informations acquises comme des savoirs, qui résonnent comme une injonction vitale et qui prévalent dans la pratique des professionnels et des patientes.

Seul le colostrum qui ne passe pas par le réfrigérateur est considéré comme «fraîchement exprimé ». D’ailleurs, rajoute Sandrine, «le colostrum, on essaie que ça ne passe pas par la case frigo » alors qu'il semble que cette étape ne pose pas de problème pour le lait. En effet, le lait cru passe de la chambre de la mère ou du réfrigérateur de la maternité à celui de la biberonnerie de la néonatologie où il est préparé - au même titre que le lait de lactarium - c'est-à-dire reconditionné et étiqueté selon les règles d'hygiènes de l'AFSSA pour être prêt à sa distribution future par la machine qui le délivre au goutte-à-goutte par sonde gastrique. Cette étape d'uniformisation le fait transiter du bac en bas du réfrigérateur où sont stockés les laits en attente aux étages supérieurs en fonction des heures prévues pour sa distribution.

Si c'est sa condition d'aliment qui lui fait passer la porte du réfrigérateur, en particulier les risques de contamination microbiologique qu'il encourt au-delà de 4 heures et les risques augmentés qu'il fait courir à ces enfants du fait de leur grande prématurité (AFSSA), c'est aussi ce franchissement qui le fait basculer du côté des médicaments et de la médication. Passé les délais qui lui sont accordés, l'heure de péremption, parce qu'il a été oublié ou parce que «personne n'a pu le descendre» (Sandrine), il devient impropre à la consommation et perd alors ses vertus thérapeutiques. Suspecté d'être matériellement impur (Douglas 1971) parce que susceptible d'être contaminé par des organismes pathogènes, il perd toute préciosité et est alors considéré comme un déchet (Frias 2004). Il est jeté.

L'anthropologie et plus largement les sciences sociales ont montré qu'allaiter est une expérience culturelle et l'allaitement, une norme sociale. Il ne s'agit pas seulement de phénomènes biologiques mais aussi et tout autant d'expériences sociales et culturelles qui engagent l'organisation socio-économique de la société, les rôles sociaux attribués à chaque sexe, des systèmes de valeurs et de croyances et des représentations symboliques du corps, de ses substances et des rapports entre elles (Cassidy \& El Tom 2014). Et comme le dévoilent les travaux de l'ANR Colostrum, si dans de nombreuses sociétés il est - ou a été (Dupont 
2002) - rejeté et considéré comme dangereux pour la santé de l'enfant ${ }^{20}$, d'autres le valorisent tant pour ses apports, en particulier immunologiques, que pour ses effets curatifs (soigner la peau, les orgelets ${ }^{21}$ ). Le colostrum n'est donc pas seulement considéré comme un aliment mais aussi comme un médicament qui peut soigner, guérir.

L'essai clinique est à l'origine d'une nouvelle forme d'allaitement maternel au sein de l'hôpital impliquant le développement de pratiques de don «artificiel» de colostrum et nécessitant son transport du sein de la mère à la bouche du nouveau-né et, parfois, sa conservation. Alors qu'il s'agit d'un protocole habituel pour le lait mature, le don «artificiel» du premier lait est une procédure thérapeutique qui a nécessité pour sa mise en ouvre l'adaptation des savoir-faire professionnels tant à la substance qu'à la multiplication et la nouveauté de sa manipulation. Le colostrum, habituellement tété donc invisible et non manipulé, transite ici par des mains et des objets. Sa prescription par ordonnance et les étapes par lesquelles il passe comme son expression assistée par les soignants, son recueil dans une seringue qui en fait « une dose», sa conservation dans le réfrigérateur prévu pour les médicaments l'inscrivent dans un circuit où le don de colostrum est assimilé à un traitement. Il subit de la part des soignants un traitement intellectuel et matériel particulier, une rationalisation scientifique et, par cette action, il bascule totalement de la sphère de l'alimentaire au domaine médical. 


\section{Notes}

1. Allemagne, Bolivie, Brésil, Burkina-Faso, Cambodge, France, Maroc.

2. http://nosobase.chu-lyon.fr/recommandations/ afssa/2005_alimentation_AFSSA.pdf.

3. Expression employée par Valérie Verrahsselt, responsable du volet immunologie au sein de l'ANR Colostrum, comme métaphore pour vulgariser les résultats de ses recherches.

4. Dans les 7 pays, 100 mères ont été interrogées par questionnaire 2 jours après l'accouchement, 20 ont été revues en entretien. De la même façon, un questionnaire a été conduit auprès de 20 membres du personnel soignant dont 4 ont accepté un entretien par la suite.

5. La présentation détaillée de l'ANR Colostrum, la problématique du programme, les carnets de terrains et l'accès aux entretiens et à leur retranscription sont disponibles sur https://colostrum. hypotheses.org/.

6. Infirmière puéricultrice, consultante en lactation au service de néonatalogie.

7. Données internes au service.

8. Pédiatre spécialisée en nutrition au sein du service de réanimation néonatale.

9. Recherche financée par le CHU de Nice.

10. La thèse de Charlotte Isnard dirigée par le Docteur Stéphanie de Smet est consacrée à cette étude. Au stade actuel de l'essai clinique, il n'est pas possible d'apporter plus de détails à propos du protocole ni de fournir les premiers résultats. Il faudra attendre la fin de l'étude et l'autorisation de la Délégation de la recherche clinique et de l'innovation du $\mathrm{CHU}$ de Nice.

11. Extrait du «projet colostrum».

12. Marie Grivel, sage-femme, a consacré son mémoire de fin d'étude à ce dernier point en 2016.
13. Autre terme employé par les soignants concernés pour parler du recueil de colostrum.

14. La vidéo de l'expression manuelle du colostrum mise en place par le Dr Jane Morton est visible à partir de ce lien: https://med.stanford.edu/newborns/ professional-education/breastfeeding/hand-expressing-milk.html.

15. La maternité de cet établissement possède le label HAB, Hôpital Ami des Bébés, initiative mise en place par l'Organisme Mondial de la Santé (OMS).

16. Ce délai est calqué sur les travaux du Dr Jane Morton.

17. De nombreux travaux en biologie comme ceux de Castellote et al. (2011) montrent que le colostrum d'une femme qui a donné naissance de façon prématurée est plus riche en immunoglobulines A (igA), lizozyme et lactoferrine mais aussi en protéines, presque deux fois plus que le lait dit mature.

18. Le protocole ne laisse pas plus de place au père - membre de la triade mère-père-enfant prise en charge par les soignants lors d'un accouchement que dans le cas d'une naissance à terme sans séparation. De la même manière qu'il donne le biberon ou aide sa femme à mettre l'enfant au sein, il peut ici participer au don de colostrum. Cependant, il s'agit là d'une nouvelle pratique de paternage.

19. Le terme n'est pas choisi au hasard. Le colostrum est souvent comparé à un vaccin ou qualifié de «premier vaccin» tant par les mères que par les soignants interrogés au cours de l'enquête ANR, en France comme dans d'autres pays.

20. Par exemple, au Burkina Faso, le colostrum est désigné par le terme kinndi en fulfudé qui signifie «quelque chose de trouble», «liquide amer» ou encore «liquide impropre».

21. Informations issues de l'enquête conduite en France.

\section{I'auteure}

Docteure en anthropologie sociale, Céline Verguet est chercheuse associée au laboratoire d'anthropologie et de psychologie cliniques, cognitives et sociales (LAPCOS, EA7278), Université Côte d'Azur.

\section{Iconographie}

Crédits de l'ensemble des images: @ ANR Colostrum sauf pour la figure 1 @ tous droits réservés. 


\section{Références}

Candau, J. et al. (à paraître) «Une approche bioculturelle du premier aliment du nouveau-né» in E. Herrscher \& I. Seguy dir. Allaitement et pratiques de sevrage. Approches pluridisciplinaires et diachroniques. Paris: Éditions de l'INED.

Candau, J. \& C. Verguet (à paraître) « Le don et la consommation néonatale du colostrum: pratiques et représentations en France» in E. Herrscher \& I. Seguy dir. Allaitement et pratiques de sevrage. Approches pluridisciplinaires et diachroniques. Paris: Éditions de l'INED.

Cassidy, T. \& A. El Tom dir. 2014 Ethnography of Breastfeeding: Cultural Contexts and confrontations. London: Bloomsbury.

Castellote, C. et al. 2011 «Premature delivery influences the immunological composition of colostrum and transitional and mature human milk», Journal of Nutrition 141 (6) : 1181-1187.

Douglas, M. 1971 De la souillure. Essai sur les notions de pollution et de tabou. Paris: François Maspero.

Dupont, F. 2002 «Le lait du père» in P. Moreau dir. Corps romains. Grenoble: Jérôme Million.
Frias, A. 2004 «La symbolique des déchets. L'impur, le sauvage, la mort » in F. Héritier \& M. Xanthakou dir. Corps et affects. Paris: Odile Jacob.

Gojard, S. 2003 «Lallaitement, une norme sociale», Spirale 27 (3) : 133-137.

Grivel, M. 2016 Recueil de colostrum chez les mères de nouveau-nés en dessous de 32 SA. Mise en place au CHU de Nice. Mémoire présenté et soutenu publiquement en vue de l'obtention du diplôme de Sage-Femme, sous la direction du $\mathrm{D}^{\mathrm{r}}$ Casagrande.

Snyder, R. et al. 2017 «Early provision of oropharyngeal colostrum leads to sustained breast milk feedings in preterm infants », Pediatrics and Neonatology May 10: $1-7$.

Sola, C. 2015 « Toucher et savoir. Une anthropologie des happerceptions professionnelles», ethnographiques. org 31, La part de la main, décembre 2015. [En ligne] : ethnographiques.org/2015/Sola.

Verguet, C. et al. 2016 «Loptimisation du recueil de colostrum et le don aux bébés prématurés: pourquoi et comment?», Cahiers de la puéricultrice 295: 16-19.

\section{Pour citer l'article}

Verguet, C. 2018 «Le don de colostrum en situation de séparation mère-enfant. De l'aliment au traitement », Techniques\& Culture 69 «Le temps des aliments», p. 168-179. 